Cahiers de recherches médiévales

\title{
La nouvelle école de philologie romane et sa perception de la littérature médiévale
}

\section{Charles Ridoux}

\section{(2) OpenEdition \\ 1 Journals}

Édition électronique

URL : https://journals.openedition.org/crm/2506

DOI : $10.4000 / \mathrm{crm} .2506$

ISSN : 1955-2424

Éditeur

Honoré Champion

\section{Édition imprimée}

Date de publication : 12 décembre 1996

Pagination : 187-198

ISSN : 1272-9752

\section{Référence électronique}

Charles Ridoux, "La nouvelle école de philologie romane et sa perception de la littérature médiévale », Cahiers de recherches médiévales [En ligne], 2 | 1996, mis en ligne le 04 février 2008, consulté le 15 décembre 2022. URL : http://journals.openedition.org/crm/2506 ; DOI : https://doi.org/10.4000/crm. 2506 


\section{【RM}

\section{La nouvelle école de philologie romane et sa perception de la littérature médiévale}

Au tournant des années 1860 apparait en France, préparée par les travaux d'érudits tels que Raynouard, Fauriel, Guessard et Paulin Paris, et formée également à l'école de la science allemande, une nouvelle école de philologie romane qui va s'imposer et se doter de ses propres organes, avec la Revue critique en 1865, la Romania en 1872 et la Société des anciens textes français, fondée en 1875 et qui édite un bulletin annuel. Les chefs de file de cette nouvelle école sont les deux directeurs de la Romania, Gaston Paris et Paul Meyer dont la collaboration durera avec constance jusqu'à la mort de Gaston Paris en 1903. Présents dans tous les hauts lieu de l'érudition - au Collège de France, à l'Ecole des chartes, à l'Ecole des Hautes Etudes, à l'Académie des Inscriptions et Belles-Lettres et notamment à la Commission de l'Histoire littéraine de la France où ils côtoient Renan et Léopold Delisle, le savant directeur de la Bibliothèque nationale - les romanistes français associent étroitement l'étude de la philologie et l'étude du passé littéraire de la France médiévale. Ce double aspect de leur activité n'est pas sans colorer la vision qu'ils ont de la littérature médiévale, les amenant à s'écarter, parfois assez sensiblement, de la plupart des jugements de leurs contemporains qui obéissent plutôt à des engagements politiques ou à des modes littéraires. On peut ainsi avancer l'idée qu'il existe un "Moyen Age des médiévistes" qu'il convient d'appréhender sur le fond des diverses images du Moyen Age véhiculées durant la seconde moitié du XIX $s$.

Trois visions globales du Moyen Age se concurrencent sur le plan idéologique: un Moyen Age «républicain»- représenté par la trilogie Michelet, Renan, Taine- qui a tendance à confondre hostilité à l'égard du Second Empire et rejet du Moyen Age, avec toutes sortes de nuances, bien entendu, et notamment une sourde nostalgie pour ces temps de solide affirmation de soi-même en contraste avec le mal du siècle moderne; en face, les catholiques se partagent en deux branches: d'une part, les «ultramontains», regroupés derrière le journal de Louis Veuillot, $L$ 'Univers, et qui célèbrent avant tout dans la Chrétienté occidentale le grand âge de la foi; et les «libéraux », représentés par Montalembert, qui trouvent dans certaines institutions médiévales des éléments précurseurs du gouvernement constitutionnel et qui associent l'Eglise catholique à la liberté pour sa résistance à l'emprise des seigneurs féodaux.

Mais le Moyen Age est aussi une référence littéraire et, dans le domaine des jugements esthétiques qu'il suscite chez les auteurs du XIX $\mathrm{f}$., il apparait comme un objet qui engendre à la fois l'intérêt et la contestation. Un premier aspect de cette ambivalence s'appuie sur le constat que "si le Moyen Age a produit en France une littérature collective vigoureuse et savoureuse, il n'a pas engendré de créateurs individuels. La perception de cette littérature est donc partagée entre l'enthousiasme pour une poésie nationale et populaire et le regret de l'absence d'écrivains de génie "' . En outre, on observe, au fur et à mesure que s'accumulent

\footnotetext{
${ }^{1}$ M.G. Glencross, Un thème médiéval dans le romantisme francais : la "matière de Bretagne » dans la critique littéraire et dans l'érudition de 1800 à 1860. Thèse de doctorat d'Université présentée à l'Université Stendhal de Grenoble, oct. 1990, dactyl., p. 287.
} 
les progrès de l'érudition, une scission entre les spécialistes de la littérature médiévale qui exhument des textes indépendamment de toute considération quant à leur valeur esthétique, et le large public, confronté à des textes qui lui paraissent souvent, de prime abord, rebutants, aussi bien du fait de leur contenu que d'une langue qui leur est inaccessible. Et les critiques littéraires, de Sainte-Beuve à Brunetière, resteront sur la réserve ou porteront un jugement sévère quant à la qualité artistique des textes mis au jour par les érudits.

Durant la seconde moitié du XIX ${ }^{\mathrm{e}}$ s., deux visions du Moyen Age exerceront une influence particulière : celle qui se dégage de la Légende des siècles de Victor Hugo, et celle que présente Leconte de Lisle. Si la seconde reprend avec virulence les attaques des Lumières contre " les siècles obscurs ", la première apparaît comme une création personnelle du poète, déconcertant de ce fait républicains et catholiques. Hugo ne saurait réduire son Moyen Age à une vision monolithique - enfer ou paradis ; c'est l'antithèse qui lui convient enfer et paradis. Mais l'aspect sinistre et lugubre du Moyen Age prédomine; c'est que Hugo «demeure un romantique, chez qui l'aspect sinistre du Moyen Age suscite un frisson esthétique plus qu'une indignation morale ${ }^{2}$. C'est tout au long de sa carrière que Leconte de Lisle témoigne d'une hostilité farouche à l'égard du Moyen Age. Le poète atteint le sommet de sa haine envers le Moyen Age dans un poème intitulé "Les Siècles maudits", datant de 1883, et qui est un véritable «compendium de tout ce que le XIX ${ }^{e}$ s. ennemi du Moyen Age a trouvé de plus sinistre pour le fustiger $"{ }^{3}$; le poème commence ainsi :

Hideux siècles de foi, de lèpre et de famine

pour se terminer sur cette malédiction:

Dans chacune de vos exécrables minutes,

O siècles d'égorgeurs, de lâches et de brutes,

Honte de ce vieux globe et de l'humanité,

Maudits, soyez maudits, et pour l'étemité !

Le rejet du Moyen Age s'exprime de façon moins violente, mais peut-être plus insidieuse, dans l'attitude de fine dérision, qui est celle d'un Théophile Gautier et des Pamassiens. On trouve, en revanche, chez Barbey d'Aurevilly, un Moyen Age autrement passionné, échevelé, de grande allure, de même que chez Huysmans ou Léon Bloy, avec toutes les outrances de rigueur chez ce pèlerin de l'absolu. De fait, le Moyen Age des poètes et des écrivains se présente avant tout selon un paradigme qui oppose modemité et tradition, chacun, au gré de ses évolutions personnelles, mettant l'accent sur l'un ou l'autre pôle; il en va ainsi des visions propres à Verlaine, d'une part, à Rimbaud, d'autre part. $\Pi$ en est, cependant, qui ne voient nul mystère dans le Moyen Age, mais plutôt un temps d'innocence naive et enfantine. C'est le cas d'Anatole France, dont un indulgent éclectisme et une fine condescendance caractérisent tous les récits médiévaux. Une telle condescendance recouvre à l'évidence le sentiment de supériorité de l'homme moderne, qui considère les «enfantillages» du Moyen Age avec le regard amusé d'un adulte raisonnable. Plus

2 J. Dakyns, The Middle Ages in French Literature 1851-1900, London, Oxford University Press, 1973, p. 141.

${ }^{3}$ bid, p. 160. 
savoureux, mais aussi plus inquiétant, est le Moyen Age de Marcel Schwob, qui était fasciné par les rapports de crimes singuliers qu'il exhumait des archives du $X v^{\mathbb{E}} \mathrm{s}$., par les criminels et les fléaux de cette époque.

Il convient de replacer ce rapide parcours des positions prises envers le Moyen Age par quelques-uns des principaux auteurs de la seconde moitié du XIX $\mathrm{X}^{\ell} \mathrm{s}$. dans le climat général des décennies 1880 et 1890 . A ce moment déjà, l'action des érudits commençait à porter des fruits dans des milieux cultivés plus vastes. Des critiques et des écrivains, tels que Jules Lemaitre ou Anatole France, connaissent et apprécient l'œuvre d'un Gaston Paris ${ }^{4}$. Jane Dakyns rapporte qu' "Anatole France lit, en 1888, le Manuel d'Ancien francais de Gaston Paris à l'ombre d'un chêne vénérable ". L'action de Gaston Paris atteint le grand public cultivé avec la publication des deux volumes de La Poésie au Moyen Age (1885-1895) et de son Manuel d'Ancien français (1888). Ce succès attire les foudres du grand chantre des études classiques, Brunetière qui proteste, dans un article violemment polémique de la Revue des Deux-Mondes contre le développement des études médiévales et fustige «cette glorification systématique de la langue et de la littérature du Moyen Age $»^{6}$. Mais l'heure n'est plus au dénigrement du Moyen Age et le goût de la légende s'en prend au positivisme ambiant. Dans son introduction aux Grandes légendes de France (1892), le critique wagnérien Edouard Schuré, pour qui "l'âme celtique était l'âme indestructible de la France », insiste sur «le pouvoir consolateur de la légende »" Une atmosphère d'idéalisme ou de mysticisme remplace le positivisme du Second Empire, dont témoignent des revues telles que Le Saint Graal d'Emmanuel Signoret (fondée en 1892) ou les deux revues catholiques belges Durendal (1894) et La Trêve-Dieu (1897), ainsi que l'immense succès obtenu par la Vie de saint François d'Assise de Paul Sabatier (1894). Dans le domaine artistique, l'heure de gloire a sonné pour les Pré-Raphaélites anglais, qui étaient toumés en dérision durant les années 1850. Gustave Moreau introduit le goût des licomes, des paons et des forêts enchantées, tandis que Richard Wagner est responsable de «tout un bric-à-brac d'olifants et d'oriflammes, de heaulmes et de hauberts, de trompettes résonnant aux quatre coins de l'horizon ${ }^{8}$.

Durant la seconde moitié du $\mathrm{XIX}^{\mathrm{e}}$ s., trois aspects de la littérature médiévale furent particulièrement mis en vedette, dépassant le cadre restreint des spécialistes pour toucher le grand public: la Chanson de Roland, le personnage et la poésie de Villon, enfin les romans arthuriens.

Sous le Second Empire, catholiques et républicains étaient unanimes dans leur enthousiasme pour la Chanson de Roland, par sentiment patriotique. Le poème est traduit en français moderne - par Delécluze en 1845, puis par François Génin en 1850 - il est qualifié de sublime en 1852 dans un article de L. Vitet paru dans la Revue des Deux-Mondes, et il est porté aux nues par l'enthousiasme de Léon Gautier qui n'hésite pas à déclarer que "la Chanson de Roland vaut l'Iliade " ${ }^{9}$. Taine et Mérimée, cependant, se tiennent à l'écart de ce

${ }^{4} \mathrm{~J}$. Lemaitre, Les Contemporains, t. 3, 1887, p. 235.

5 J. Dakyns, op. cit., p. 208.

${ }^{6}$ Brunetière, Revue des Deux-Mondes, ler juin 1879.

${ }^{7}$ J. Dakyns, op. cit. p. 210.

${ }^{8}$ Ibid, p. 265.

${ }^{9}$ L. Gautier, Les Epopées francaises, t. 1, 1865, p. 656. 
concert de louanges. Mais c'est la crise de 1870-1871 qui va porter Roland aux premières lignes du combat pour la réforme intellectuelle et morale du pays après le désastre dans lequel s'est englouti l'Empire. Selon le propos de Léon Gautier : « Il a fallu la guerre de 1870 pour nous en donner l'intelligence et l'amour. Sedan a fait comprendre Roncevaux $»^{10}$. En 1878, la Chanson de Roland obtint les honneurs de l'agrégation, et la même année fut érigée sur le parvis de Notre-Dame la colossale statue de Charlemagne par Roche.

Si la haute figure de Roland était attachée aux préoccupations patriotiques, le personnage de Villon était l'idole des républicains. L'édition de ses œuvres par Paul Lacroix en $1854-$ la première depuis celle de Prompsault (1832) - fut suivie par celle de Jannet en 1867. Et la première étude sur la biographie sortit en 1859, avec la thèse de Campaux en Sorbonne. Gautier avait donné l'image d'un Villon subversif et sentimental dans Les Grotesques et Banville composa durant les années 1860 ses Trente-Six Ballades joyeuses à la manière de François Villon (publiées collectivement en 1873). L'enthousiasme des républicains à l'égard de Villon répondait à leur attrait pour les œuvres satiriques du Moyen Age en général, le Roman de la Rose de Jean de Meung, le Roman de Renart ou les fabliaux, «en tant qu'œuvres dépositaires de l'esprit gaulois" ". Toutefois, ni Michelet ni Renan ne partagèrent ce goût.

Aux antipodes de "l'esprit gaulois», la légende arthurienne séduit par sa puissance d'imagination. Elle inspire Quinet dans son Merlin l'Enchanteur (1860); l'auteur déclare dans sa préface avoir voulu «renouveler l'imagination française dans les sources nationales $" 12$. Durant le Second Empire, la légende arthurienne fut popularisée par les travaux d'érudits tels que Théodore Hersart de La Villemarqué ou Paulin Paris. Quant à l'historien Henri Martin, il "s'efforçait de persuader ses lecteurs du caractère prérévolutionnaire de la légende arthurienne $\aleph^{13}$. Il oppose le chevalier arthurien, fils des Gaulois, au chevalier carolingien, fils des Francs et il loue la nature «démocratique » de la Table Ronde. Certains, en revanche, dénoncent la Table Ronde pour être contraire à la morale, tel le catholique Arbois de Jubainville, qui déplore "cette prédominance de l'élément érotique qui distingue les romans de la Table Ronde de ceux du Cycle carlovingien $»^{14}$.

Ainsi, le Moyen Age, qui fut tout au long du siècle un des lieux de prédilection autour duquel se menèrent les affrontements idéologiques suscités par la fracture consécutive à la Révolution, servit également de référence littéraire à toutes les écoles qui se succédèrent sous le Second Empire et dans les débuts de la III République. Dans les deux cas, le Moyen Age suscita généralement des sentiments d'enthousiasme ou de rejet marqués; rares furent ceux qui gardèrent à son égard une attitude sereine. Même dans le public cultivé, les jugements étaient fondés sur une connaissance très relative des œuvres de la littérature médiévale; ce $n^{\prime}$ 'est que vers la fin du XIX $\mathbf{e}$. que l'imposant travail d'édition des textes par les érudits allait porter ses fruits, et encore la difficulté de la langue constituera longtemps une barrière, jusqu'au jour où, dans les trois dernières décennies du $\mathrm{xx}^{\mathrm{e}} \mathrm{s}$., les traductions iront se

\footnotetext{
${ }^{10}$ L. Gautier, Les Epopées françaises, t. 2, 1892, p. 725.

${ }^{11}$ Ibid, p. 102.

${ }^{12}$ E. Quinet, Euvres complètes, t. 16, p.v.

${ }^{13}$ Ibid, p. 105.

${ }^{14} \mathrm{H}$. d'Arbois de Jubainville, Revue des questions historiques, t. 6, 1er avril 1869, p. 527.
} 
multipliant. On peut se demander quelles furent alors les prises de position de ces érudits qui, eux, avaient accès aux textes, s'ils partagèrent ou non les passions idéologiques et esthétiques de leurs contemporains, et quel fut le jugement qu'ils portèrent sur les divers genres et sur les œuvres du Moyen Age. En un mot, y a-t-il eu un «Moyen Age des médiévistes " ?

Dans l'Avertissement du tome XIX de l'Histoire littéraire de la France paru en 1838, volume où il fut question d'Aucassin et Nicolette, de Partanopeus de Blois et de Marie de France, la Commission qui poursuit l'œuvre des Bénédictins de Saint-Maur s'exprime en ces termes à propos de la littérature médiévale en français :

Nous donnons des extraits de ces productions du Moyen Age, non certes dans le dessein de les proposer pour modèles, mais parce qu'elles sont des faits qui appartiennent à l'histoire littéraire des Français et parce qu'elles contribuent à montrer à quel degré de puérilité et de barbarie les talents peuvent descendre lorsqu'ils n'ont pas commencé, et plus encore, lorsqu'ils cessent de suivre les routes tracées par les grands maitres de l'art des vers. ${ }^{15}$

Le mépris hautain, hérité de l'âge classique, va bientôt disparaitre, sauf dans certaines citadelles de l'esprit irréductibles à tout ce qui n'entre pas dans le canon du classicisme. Mais il restera, et chez ceux-là mêmes qui consacrent le meilleur de leur vie à l'étude des œurvres de la littérature française du Moyen Age, cette approche- que nous appellerons documentaire - qui consiste à s'intéresser aux textes en tant qu'ils représentent un état des moeurs de nos ancêtres médiévaux sans se soucier de leur valeur esthétique. Cette approche documentaire, nous semble-t-il, est la caractéristique dominante de l'attitude des érudits médiévistes à l'égard de leur objet d'étude. Michael Glencross estime que, de 1800 à 1860 , l'essentiel des efforts a porté sur l'intégration de l'époque médiévale dans l'histoire littéraire de la France : «Il ne s'agit pas d'une revalorisation esthétique des textes mais d'une tentative largement réussie d'élargir le champ de l'histoire litteraire ${ }^{16}$. Cette tendance demeure encore largement valable jusqu'à la fin du siècle, si ce n'est jusqu'au sortir de la Première Guerre mondiale. A cela s'ajoute, surtout à partir des années 1830 et plus encore durant le Second Empire et après 1870, la note de patriotisme national qui marque l'activité des érudits.

L'attitude des pères fondateurs du médiévisme ne sera au fond pas très différente. Dans l'un de ses premiers discours devant la Société des anciens textes français, Gaston Paris se fait l'avocat d'une approche neutre, sans exclusive, des textes médiévaux: "Nous ne ferons aucune exclusion systematique. Ecarter tel genre parce qu'il est ennuyeux, tel autre parce qu'il heurte le sentiment du decorum que nous devons à une éducation supérieure, ce serait se condamner à voiler bien des aspects de la vie de nos ancêtres ${ }^{17}$. Gaston Paris admet une infériorité du Moyen Age par rapport à l'antiquité tant dans le domaine esthétique que dans celui des idées, mais il souligne la proximité de cette époque avec les temps modernes et il conclut par cette formule: "Nous n'avons ni à l'exalter, ni à le rabaisser, mais à le connaître ${ }^{18}$. Le savant médiéviste revient sur cette question devant le même public l'année

\footnotetext{
${ }^{15}$ "Avertissement ", HLF, 19, 1838, p. xiii.

${ }^{16} \mathrm{~J}$. Glencross, op. cit., p. 237.

${ }^{17}$ Bulletin SATF, 2, 1876, p. 51.

${ }^{18}$ Ibid, p. 52.
} 
suivante, brossant un tableau des divers genres qui remportent du succès auprès du public moderne et concluant sur deux façons de comprendre le Moyen Age, selon deux veines, l'une sérieuse et pieuse, l'autre comique et satirique:

Les uns s'exaltent à la lecture des chansons de geste, s'édifient dans les pieuses légendes, si naivement conçues at contées, pleurent aux mystères où se déroulent en scènes infiniment diverses la vie du Christ ou des saints; les autres prennent plaisir aux contes bourgeois, dont le style leste et piquant, dont surtout la vieille langue fait pardonner la grossièreté, rient aux farces vivantes et joyeuses qui ont préludé à notre grand théâtre comique, et s'amusent des mordantes satires lancées à toute volée, sans trève ni répit, contre tout ce qui constituait jadis les institutions établies et ceux qui les représentaient. ${ }^{19}$

Entre ces deux approches, l'érudit refuse de choisir, tirant la conclusion que la tâche de l'heure est de publier sans arbitraire tous les monuments légués par le Moyen Age. Gaston Paris, polémiquant cette fois avec Brunetière qui s'était insurgé dans la Revue des DeuxMondes contre les succès de la littérature médiévale, précise une fois de plus l'objectif qui est assigné à la Société des anciens textes français: "L'esprit qui anime la Société des anciens textes français est, nous ne saurions trop le redire, purement historique. Nous ne prétendons ni dénigrer ni réhabiliter le Moyen Age, ses institutions, ses croyances, ses mœurs, sa langue et sa littérature, nous prétendons le faire connaitre et le faire comprendre $»^{20}$. Mais il insiste ici sur un thème qui lui est particulièrement cher, qui est le culte des ancêtres:

Quoi de plus intime dans cette histoire que l'étude des façons de parler, de penser et de sentir de nos vieux pères? Gràce aux anciens textes, bien publiés et bien commentés, nous revivons de leur vie, nous savons ce qui les intéressait, ce qui les passionnait, ce qui les divisait, ce qui les faisait pleurer et rire; nous retrouvons leurs croyances, leurs moeurs, leurs habitudes d'esprit; nous pouvons presque, armés des méthodes pénétrantes et rigoureuses de la science modeme, entendre leurs voix éteintes depuis des siècles. ${ }^{21}$

Ici, le savant se fait lyrique et, à côté de «l'écho vibrant " des paroles des ancêtres, il évoque le « fragile parchemin » qui en a recueilli la trace. L'émotion qui l'anime alors lui fait franchir sans effort l'abîme qui sépare la lettre morte de la parole vivante. A cet égard, sans doute, Gaston Paris, qui apparait généralement soucieux d'une approche neutre, documentaire, n'est pas très éloigné du désir exprimé par un Michelet de "ressusciter " le passé. Et pour l'un comme pour l'autre, ce passé, c'est avant tout celui de «nos pères», cette chaîne de filiation qui nous relie à un passé médiéval qui, somme toute, n'est pas si lointain.

Il arrive parfois à Gaston Paris de porter des jugements personnels sur la valeur littéraire de telle ou telle œuvre. Ainsi, lorsqu'il présente le bilan de l'activité de son père dans la Commission de l'Histoire littéraire de la France, Gaston Paris brosse au passage, en quelques traits suggestifs, les portraits littéraires de Jean Bodel, d'Adam de la Halle, de Rutebeuf et d'Adenet le Roi. Pour ce dernier, il juge que le roman de Cléomadès est la meilleure de ses cuvres: «Les petits vers accouplés convenaient mieux que les grandes laisses monorimes des chansons de geste à sa facilité un peu banale, comme les aventures

\footnotetext{
${ }^{19}$ Bulletin SATF, 3, 1877, p. 57.

${ }^{20}$ Bulletin SATF, 5, 1879, p. 50.

${ }^{21}$ Ibid, p. 50.
} 
romanesques allaient mieux à son imagination que les simples récits et les continuelles batailles de nos vieux poèmes nationaux ${ }^{22}$.

La prédominance de l'approche documentaire est commune aux deux maitres de la nouvelle école, Gaston Paris et Paul Meyer. Mais ce dernier ne manque pas d'exprimer ses jugements personnels sur les cuvres proposées chaque année aux assemblées de la Société des anciens textes français; le rapport du secrétaire, que Paul Meyer fournira avec constance depuis la fondation de la Société jusqu'à la guerre de 1914 est une mine de renseignements sur ses goûts littéraires et fait nettement apparaître en ce domaine une compétence égale à celle qu'il avait acquise sur le plan linguistique ou historique, ainsi qu'un jugement clair et souvent plein de finesse. Une particularité propre, semble-t-il, à Paul Meyer est son intérêt marqué pour la fin du Moyen Age. Il constate, en 1880, que les études médiévales, tant en France qu'à l'étranger, ont eu tendance à laisser cette période de côté: «L'étude de nos vieux auteurs, poussée depuis quelques années avec tant d'ardeur en France, en Allemagne et en Italie, s'est principalement portée vers la période la plus ancienne de notre littérature, de sorte qu'il reste probablement plus de découvertes à faire pour le $X \mathrm{XI}^{\varepsilon}$ et le $\mathrm{XV}{ }^{\varepsilon} \mathrm{s}$. que pour les temps antérieurs ${ }^{23}$. Il revient sur ce thème en 1888 , précisant alors l'intérêt que présente à ses yeux la littérature de cette période :

Elle offre pourtant, en son extrême variété, un bien vif intérêt, soit qu'elle continue la tradition des siècles précédents, soit qu'elle présage la Renaissance. Elle a surtout, pour le chercheur, cet attrait incomparable que, gráoe à la richesse des archives de cette époque, il est le plus souvent possible de trouver pour chaque auteur des documents qui nous renseignent sur l'époque où il vivait, sur le milieu dans lequel il a exercé ses facultés litéraires, au lieu qu'au XIIf $s$., par exemple, et plus encore au Xú $\mathrm{s}$., des ouvrages répandus, célèbres même, nous sont parvenus anonymes ou sous des noms auxquels nous ne pouvons joindre aucune notion biographique ${ }^{24}$

A nouveau, ce qui se manifeste ici, c'est l'intérêt pour l'histoire, pour l'approche documentaire.

Beaucoup plus rares, parmi les médiévistes, sont les témoignages de sympathie pour le Moyen Age considéré comme un "Age de Foi». Parmi ceux-ci, Petit de Julleville, dont Gaston Paris nous apprend, dans l'éloge qu'il en fait devant la Société des anciens textes français qu' « il n'étudiait pas le Moyen Age uniquement, ou tout à fait, avec la pure curiosité d'un savant ou l'intérêt d'un descendant soucieux de connaitre ce qu'ont été ses aïeux : il éprouvait pour cette époque de foi une sympathie particulière, qui tenait à ses convictions personnelles, et qui a pu quelquefois influencer ses jugements, sans jamais cependant l'entraîner à des exagérations que son goût délicat aurait réprouvées ou à des réticences que sa probité littéraire ne lui aurait pas permises ${ }^{25}$. Une telle réserve n'était certes pas dans le tempérament du bouillant Léon Gautier qui voua toute sa carrière à la défense du Moyen Age. Pour lui, qui disait à propos de La Sorcière de Michelet: "Ce livre est l'histoire de Satan, écrite sous sa dictée ${ }^{26}$, il était impensable de dissocier véritablement jugement

${ }^{22}$ G. Paris, « Notice sur Paulin Paris (mort le 13 février 1881) », HLF, 29, 1885, p. xii.

${ }^{23}$ Bulletin SATF, 6, 1880, p. 101.

${ }^{24}$ Bulletin SATF, 14, 1888 , pp. $52-53$.

${ }^{25}$ Bulletin SATF, 26, 1900 , p. 42.

${ }^{26} \mathrm{~L}$. Gautier, Etudes littéraires pour la défense de l'Eglise, 1865, p. 149. 
esthétique et jugement moral. Au demeurant, cette partialité assumée avait pour elle le bénéfice de la franchise et de la sincérité.

Quand les médiévistes de l'époque se risquent à prononcer des jugements à propos d'un genre ou d'une curve de la littérature médiévale, ces jugements ne portent pas uniformément sur tous les genres et sur n'importe quelle ouvre : certains attirent davantage l'attention, suscitent des réactions plus vives, cristallisent le blâme ou l'éloge. Ces prises de positions sont parfois la conséquence d'un parti pris régionaliste, provençal chez les uns, celtisant pour d'autres. C'est ainsi que l'historien Henri Martin tient à démontrer que la conception de l'amour propre à la poésie chevaleresque est un pur produit du génie celtique. Parmi les romans arthuriens, sa prédilection se porte sur le roman de Tristan en vers, qu'il préfère aux romans de Chrétien de Troyes. Selon Michael Glencross, Martin «témoigne de l'évolution du goût de son siècle qui va vers la valorisation progressive du couple uni par le boire amoureux ${ }^{27}$. Toutefois, à la différence de Francisque Michel, Martin ne fait pas la distinction entre le texte de Béroul et celui de Thomas d'Angleterre, croyant qu'il s'agit de deux fragments d'un même poème. Farouche défenseur, au contraire, des traditions littéraires du Midi de la France, Mary-Lafon, auteur d'une Histoire politique, religieuse et littéraire du Midi de la France publiée entre 1842 et 1844, est tellement aveuglé par son parti-pris méridional qu'» il s'ingénie à faire de tout roman méridional du Moyen Age un chef-d'œuvre de la littérature mondiale » et que, publiant en 1856 une traduction abrégée des Aventures merveilleuses de Jaufre ou Geoffroi, il en fait dans son introduction « le plus beau fleuron de la couronne poétique du Moyen Age ${ }^{28}$.

La légende de Tristan semble soulever parmi les érudits du $\mathrm{XLX}^{e} \mathrm{~s}$. autant de passion qu'au temps de Chrétien de Troyes; elle donne lieu à plusieurs jugements sévères à cause de son amoralité. Sous la Monarchie de Juillet déjà, le romantique libéral Etienne Delécluze, auteur de Roland ou la chevalerie (1845), s'évertue à flétrir le cycle arthurien pour son esprit de galanterie. C'est que la critique de cette époque se fonde désormais sur des valeurs bourgeoises et non plus aristocratiques: «Le culte des valeurs chevaleresques tant prisé par la morale aristocratique de la Restauration semble exercer peu d'attrait sous la Monarchie de Juillet. Comble de l'ironie, la défense de la littérature arthurienne est assurée non pas par un critique libéral et républicain comme Delécluze mais par un érudit légitimiste et catholique comme Paulin Paris. ${ }^{29}$ "Dans le même temps, l'érudit Amaury Duval, responsable de l'Histoire littéraire de la France, se croit tenu de pourfendre des textes qui bafouent toutes les valeurs bourgeoises du $\mathrm{XIX}^{\mathrm{e}} \mathrm{s}$. :

Il faut convenir que la lecture de ces fragments de poëmes nous donne une très-désavantageuse idée des moaurs du temps où l'on se plaisait à les écouter, et sans doute à y applaudir. Pas une expression de blâme pour la femme adultère et son complice; l'un et l'autre sont préconisés, admirés pour leur beauté et leur esprit; le mari outragé, au contraire, est toujours ridiculisé, honni, conspué; ceux qui voudraient protéger son honneur, le défendre des embûches qu'on ne cesse de dresser sous ses pas, sont des perfides, des méchants, des démons, etc. ${ }^{30}$

${ }^{27} \mathrm{~J}$. Glencross, op. cit., p. 166.

${ }^{28}$ Ibid., p. 173.

${ }^{29}$ Ibid. p. 180.

${ }^{30}$ Histoire littéraire de la France, t. xix, 1838, p. 703. 
La nouvelle école évitera en général ces critiques qui reposent sur une projection des moeurs et des critères moraux de la société du XIX $\mathrm{s}$. sur d'autres temps. Sur le plan esthétique, toutefois, il n'est pas rare que soient portées de vives critiques relatives à l'absence de composition des œuvres médiévales, à leur invraisemblance ou leur facticité. Les romans de la Table Ronde, en particulier, sont visés par ces attaques. Ainsi, dans la préface à l'édition du Merlin, Gaston Paris déclare, évoquant la triple aventure de Gauvain, Tor et Pellinor: «Nous avons là des spécimens des productions ordinaires de la pauvre imagination des auteurs de romans en prose: l'invraisemblance y est poussée jusqu'à l'absurde, sans réussir à piquer la curiosité, et l'absence presque complète de motifs pour les actions les plus graves fait de tous les personnages des mannequins qui n'ont rien de vivant $»^{31}$. Dans sa leçon d'ouverture au Collège de France, le même savant qualifie les romans de la Table Ronde de "compositions factices", qui «reflètent l'idéal particulier, souvent bizarre et toujours très conventionnel, d'un monde aristocratique à la fois naïf et raffiné»; il voit en eux les ancêtres d'une litterature idéaliste qui s'est répandue dans toute l'Europe et qui a contribué à « entourer le Moyen Age de cette auréole de galanterie et de chevalerie aventureuse sous laquelle ses traits véritables ont été parfois méconnus " ${ }^{32}$.

Paul Meyer évoque lui aussi, avec un certain mépris ces « contes merveilleux» mis à la mode par les romans d'aventure, mais il n'apprécie guère davantage les « récits fastidieux de fabuleuses batailles entre Chrétiens et Sarrazins qui occupent une si grande place dans nos anciens poèmes » et il loue Raoul de Cambrai d'échapper à cette tendance pour exposer des questions juridiques sérieuses relatives au droit féodal. Présentant le Roman de Troie devant l'assemblée de la Société des anciens textes français, Paul Meyer estime cependant les aventures des héros de la Table Ronde plus «amusantes» que «les éternels combats des Grecs et des Troiens, des partisans d'Etéocle et de ceux de Polynice " ${ }^{33}$. Il explique la raison de ce succès en invoquant la culture des clercs de cette époque, tout imprégnée de la lecture des classiques latins de l'antiquité et dont la curiosité était excitée par tout ce qui touchait aux anciens héros. Cette impression de lassitude que suscitent souvent les œuvres du Moyen Age quand on les aborde sur le plan thématique - et qui marquera si profondément l'étude d'un Italo Siciliano sur Villon - Arbois de Jubainville l'exprime déjà dans son compte rendu des Anciens Poètes de la France en 1862:

Disons-le: la lecture des huit poèmes a, comme passe-temps, un véritable attrait; cependant, quand on en lit plusieurs de suite, la répétition des mêmes aventures finit par fatiguer; ces combats sans fin nous procurent moins de plaisir qu'à des chevaliers du Moyen Age dont ils peignaient la vie; cette Sarrasine qui devient chrétienne par amour, sauve les prisonniers chrétiens et se fait baptiser par immersion, vient trop souvent nous montrer, par son exemple, quelles raisons ont, dans l'administration du sacrement du baptême, décidé l'Eglise à supprimer l'immersion vers la fin du douzième siècle, pour ne conserver que l'aspersion, aujourd'hui

\footnotetext{
${ }^{31} \mathrm{G}$. Paris e J. Ulrich, Merlin. Roman en prase $d u X I I{ }^{\circ}$ s., publié d'après le ms. appartenant à $\mathrm{M}$. Alfred Huth, 2 vol., Paris, Didot, 1886.

${ }^{32}$ G. Paris, "Paulin Paris et la littérature française du Moyen Age. Leçon d'ouverture du cours de langue et littérature françaises du Moyen Age au Collège de France, le jeudi 8 décembre 1881 », Rom.,11, 1882, pp. 1-21.

${ }^{33}$ Bulletin SATF, 17, 1891, p. 50.
} 
seule en usage. De même l'exagération du merveilleux choque plus d'une fois nos habitudes modernes. ${ }^{34}$

Lassé par ce ressassement des mêmes thèmes, le critique retrouve de l'intérêt à ces textes dans leur aspect documentaire, sur le double plan de la vie et des mœurs du Moyen Age et de l'étude de la langue. Le savant celtisant partage, lui aussi, l'approche documentaire de ses confrères spécialisés dans le domaine de la littérature française. Rendant compte vers la même époque du roman en vers, datant du XIV ${ }^{e}$ s., de Girant de Roussillon, édité par Mignard en 1858, Paul Meyer juge, d'une façon générale, le XIV ${ }^{\star}$ s. comme « une époque de décadence complète pour notre vieille littérature » et il estime que ce roman, en particulier, qui n'est pour le fond que la paraphrase d'un texte latin, n'a dans la forme «rien de l'élégance, un peu stérile parfois, des trouvères du siècle précédent ${ }^{35}$. Ce qui, en fin de compte, retient l'attention du médiéviste, c'est l'intérêt d'un état de langue, qui est celui du moyen-français. Même dans le domaine de la chronique, il arrive à certains critiques de signaler l'ennui que leur cause la lecture de tel ou tel auteur. C'est ainsi que Bourciez ne supporte pas la lecture de Georges Chastellain: "Chastellain est un des plus ennuyeux écrivains que je connaisse. (...) Je veux bien que Chastellain ait eu une moralité politique supérieure à celle de Commines ; mais comme écrivain ou même comme penseur, je doute un peu qu'il puisse en être rapproché $"{ }^{36}$.

Toutefois les éloges envers des œuvres du Moyen Age ne manquent pas, en particulier dans les présentations faites par Paul Meyer à ses collègues de la Société des anciens textes français. Tout en reprenant, à propos du Méliador de Froissart, dont le manuscrit avait été retrouvé par Auguste Longnon, la critique habituelle du manque d'originalité, Paul Meyer trouve du charme et de l'intérêt à ce roman et il se réjouit de disposer avec cette œuvre d'un exemplaire qui permette d'«étudier les formes dernières des lieux communs dont se composent généralement les romans de cette classe ${ }^{37}$. Deux ans plus tard, Paul Meyer précise sa pensée: «Méliador n'est pas un chef-d'œuvre: ce n'est même pas l'œuvre la meilleure de Froissart. (...) Au point de vue littéraire, il sera curieux d'étudier les procédés de composition du poète, de chercher ce qu'il a emprunté aux anciens romans de la Table Ronde: peut-être en avait-il lu que nous ne connaissons plus ou que nous connaissons mal ${ }^{38}$. Cette fois, l'intérêt documentaire de l'œuvre ne porte pas tant sur la société médiévale que sur l'évolution littéraire des romans de la Table Ronde. Le passage d'un jugement purement historique à un jugement littéraire nous semble digne d'être relevé. II témoigne qu'après quarante ans d'efforts pour imposer la connaissance des œuvres de

\footnotetext{
${ }^{34} \mathrm{H}$. d'A. de J. : Les Anciens Poëtes de la France, publiés sous la dir. de M. Guessard, BEC, 23, 1862, p. 362.

${ }^{35} \mathrm{P}$. Meyer, $\mathrm{Cr}$ de Le Roman en vers de très-excellent, puissant et noble homme Girart de Rossillon, jadis duc de Bourgoigne, publié pour la première fois d'après les manuscrits de Paris, de Sens et de Troyes, avec de nombreuses notes philologiques et neuf dessins, dont six chromo-lithographiés, suivi de l'Histoire des premiers temps feodaux, par Mignard, Paris et Dijon, 1858, BEC, 22, 1861, p. 186190.

${ }^{36}$ E. Bourciez, cr de G. Pérouse, Georges Chastellain, Paris, Champion, 1910, Revue Critique, 442, 1910, p. 492.

${ }^{37}$ Bulletin SATF, 24, 1898 , p. 56.

${ }^{38}$ Bulletin SATF, 26, 1900, p. 51.
} 
l'ancienne littérature française, le temps commence à venir où les études vont pouvoir s'orienter sur les procédès de l'art littéraire.

Il peut être intéressant de connaître le jugement - mitigé - de Paul Meyer sur Guillaume de Machaut, puisque ce dernier appartient à cette époque de la fin du Moyen Age sur laquelle Paul Meyer souhaitait attirer l'attention des chercheurs. Paul Meyer commence par regretter que Guillaume de Machaut s'inspire par trop de la tradition de Guillaume de Lorris et que sa poésie soit trop conventionnelle. II apprécie, cependant, des digressions telles que la description de la peste de 1349 dans le Jugement du mi de Navarre ou les exempla dont il parsème ses dits. La lecture des poésies lyriques de Machaut, publiées par un savant russe, Chichmarev, n'emballent pas le critique français, qui conclut par un éloge très mesuré: "J'espère que la musique, dont il est aussi l'auteur, vaut mieux que les paroles, qui sont trop souvent monotones et banales. Guillaume de Machaut est surtout un habile versificateur et un agréable narrateur ${ }^{39}$. Le jugement de Paul Meyer sur Eustache Deschamps, dont une édition complète était en cours à l'instigation du marquis de Queux de Saint-Hilaire, apparait curieusement plus favorable: «De ce poète, rien n'est à rejeter. Par l'élégance de la diction, il vaut Guillaume de Machaut; par la variété des sujets, par la vivacité de l'esprit, il laisse bien loin derrière lui tous ses contemporains ${ }^{40}$. Toutefois, une quinzaine d'années plus tard, Paul Meyer estime que Deschamps n'est pas un grand poète et qu'il est inférieur à Christine de Pisan; il reconnaît néanmoins au poète de présenter un intérêt documentaire : «Il nous fait pénétrer plus profondément dans la connaissance de la société de son temps ${ }^{41}$. Au moment de la parution du tome IX des poésies d'Eustache Deschamps, qui contient le Miroir de Mariage, Paul Meyer modifie quelque peu son jugement, comme surpris par le ton chagrin de cette œuvre: "C'est bien l'œuvre d'un esprit vieilli et chagrin. Les idées qu'exprime Deschamps sur le mariage sont celles qui avaient cours parmi les lettrés de son temps. Mais, tandis qu'en général elles se traduisent par des scènes d'où l'élément comique n'est point entièrement banni, chez Deschamps elles sont empreintes d'une amertume profonde $~^{42}$. Une dernière fois, Paul Meyer revient sur le cas d'Eustache Deschamps, à l'occasion du commentaire fourni par Gaston Raynaud dans le tome XI des œuvres complètes du poète. Cette fois, c'est avant tout la biographie de Deschamps et la connaissance du milieu, littéraire et politique, dans lequel il a vécu qui passionne le savant médiéviste. Sans s'interdire des jugements esthétiques souvent perspicaces, Paul Meyer, on le voit, se range parmi la cohorte de ses confrères en privilégiant l'approche documentaire des œuvres médiévales.

La double polarité de l'activité des romanistes français - vers la philologie pure et vers l'histoire littéraire - se traduit de façon différenciée dans les revues où ils s'expriment ordinairement. Si l'Histoire littéraire de la France apparait comme une sorte de haut donjon de l'érudition, insensible, apparemment, aux fluctuations du temps dans sa majestueuse et lente progression au long des $\mathrm{XI}^{e}, \mathrm{XW}^{e}$ et $\mathrm{XIV}^{e}$ s., si la Romania favorise davantage le pôle philologique, les jugements de valeur sur les œuvres s'expriment davantage dans les courtes notices de la Revue critique ou dans les brèves présentations des œuvres en cours d'édition à

\footnotetext{
${ }^{39}$ Bulletin SATF, 35, 1909, p. 98.

${ }^{40}$ Bulletin SATF, 2, 1876, p. 48.

${ }^{41}$ Bulletin SATF, 18, 1892, p. 54

${ }^{42}$ Bulletin SATF, 21, 1895, p. 54
} 
la Société des anciens textes français par son secrétaire, Paul Meyer. Quant à la revue Le Moyen Age, fondée en 1888, elle se caractérise par la qualité de ses états de telle ou telle question d'histoire littéraire, souvent dus à la plume de Gédéon Huet au début de notre siècle. Finalement, l'approche documentaire dominante chez les pères fondateurs du médiévisme laissera des traces profondes dans les études médiévales au $\mathrm{XX}^{\mathrm{e}} \mathrm{s}$., et c'est surtout après la Seconde Guerre mondiale, et particulièrement après le tournant des années soixante, que diverses approches plus nettement esthétiques seront proposées pour la lecture des œuvres du Moyen Age.

Charles Ridoux (Université de Valenciennes) 